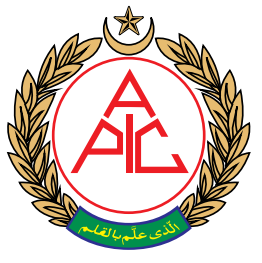

Department of Anesthesiology and Critical Care, Kermanshah University of Medical Sciences, Kermanshah, Iran

${ }^{2}$ Department of Anesthesiology and Critical Care, Kermanshah University of Medical Sciences, Kermanshah, Iran

${ }^{3}$ Imam Reza Teaching Hospital, Kermanshah University of Medical Sciences, Kermanshah, Iran

${ }^{4}$ Department of Pharmaceutics, Kermanshah University of Medical Sciences, Kermanshah, Iran

${ }^{5}$ Anesthesia

University of

Medical Sciences,

Kermanshah, Iran

Correspondence: Dr. Mitra

Yari, Department of

Anesthesiology and Critical

Care, and Imam Reza

Teaching Hospital,

Kermanshah University of

Medical Sciences,

Kermanshah, Iran.

Tel: +989181314380

E-mail:

maryamyari@protonmail.com

Received: 26 February 2020

Reviewed: 20 April 2020

Revised: 18 April 2020

Accepted: 28 April 2020

\section{A comparative study of the effects of tramadol and lidocaine gel on complications of orotracheal intubation}

\author{
Hossein Farzam ${ }^{1}$, Mitra Yari ${ }^{2,3^{*}}$, Ghobad Mohammadi ${ }^{4}$, \\ Maryam Rahmatinejad ${ }^{3,5}$
}

\section{ABSTRACT}

Background \& Objectives: Endotracheal intubation (ETI) in usually required during general anesthesia, but has some complications associated with it. Prevention of these complications is essential for patient satisfaction. This study aimed to compare the effects of topical lidocaine gel and tramadol gel on complications of ETI.

Methodology: In this clinical trial, 200 patients scheduled for laparoscopic cholecystectomy were randomly divided into five groups. The endotracheal tube cuffs were applied different gels in the following order: Group P with $1 \mathrm{ml}$ of a neutral gel, Group T1 with $1 \mathrm{ml}$ of $2.5 \%$ tramadol gel, Group T2 with $1 \mathrm{ml}$ of $5 \%$ tramadol gel, and Group L with $1 \mathrm{ml}$ of $2 \%$ lidocaine gel. In the fifth group (Group C) no gel was used. At the end of the anesthesia, the incidence of bucking, coughing, laryngospasm and/or bronchospasm, and the intensity of sore throat were recorded and compared. Data were analyzed using SPSS 20 and Chi-square, ANOVA and Kruskal-Wallis tests. The significance level of tests was considered as $p<0.05$.

Results: The use of $5 \%$ tramadol gel and lidocaine gel reduced the incidence of coughing $(p=0.004)$, but there was no significant difference between these two kinds of gels $(p=0.108)$. No cases of laryngospasm were observed. There was no significant difference between the incidence of sore throat, bucking, and bronchospasm in five groups $(p>0.05)$.

Conclusion: According to the results of this study, it seems that $5 \%$ tramadol gel decreases the incidence of coughing caused by the ETI and its impact rate is the same as with $2 \%$ lidocaine gel. But both gels don't have a significant effect on the incidence of bucking, sore throat, and bronchospasm.

Key words: Endotracheal tube; Tramadol; Lidocaine; gel; Cough

Citation: Farzam H, Yari M, Mohammadi G, Rahmatinejad M. A comparative study of the effect of tramadol and lidocaine gel on complications of endotracheal intubation. Anaesth. pain intensive care 2020;24 (2):183-188

DOI: https://doi.org/10.35975/apic.v24i2.1260

\section{INTRODUCTION}

Endotracheal intubation (ETI) during general anesthesia is associated with some complications e.g., hemodynamic changes, increased intracranial pressure, increased intraocular and blood pressure, and decreased arterial oxygenation and the anesthesiologists make every effort to prevent these to ensure patient satisfaction. ${ }^{1}$ Coughing and bucking at the end of anesthesia and at extubation are other worrisome complications. Sore throat after ETI 


\section{A comparative study of the effects of tramadol and lidocaine gel on complications}

is a common side effect. To reduce these effects, different methods and drugs have been used. $^{2}$

Several studies have evaluated different methods to reduce the complications of ETI, but no study has examined the effect of application of tramadol gel to the endotracheal tube (ETT) cuff for this purpose, although

many studies suggested local anesthetic effects of tramadol. ${ }^{1,2,3}$ The lidocaine gel has been the most commonly used gel on the cuff, but has been associated with systemic effect similar to its injectable form, ${ }^{4}$ but in the case of tramadol this issue has not been proven. ${ }^{3}$

We aimed to investigate the local effect of tramadol gel applied to the ETT cuff in decreasing the complications of ETI and compare it with placebo, lidocaine gel and with the control group.

\section{METHODOLOGY}

The study was approved by the Ethics Committee of Kermanshah University of Medical Sciences (KUMS.REC.1394.16). It is a randomized, double-blind clinical trial study. Convenient sampling technique was used. The sample was chosen from patients who were candidate for laparoscopic cholecystectomy surgery and referred to Imam Reza Hospital in 2015 .

The study protocol was registered at the Iranian Registry of Clinical Trials website (IRCTID: IRCT201507114938N3 (. It was a prospective study, carried out over one year at Imam Reza Teaching Hospital, Kermanshah, Iran.

Inclusion criteria were 20-60 y age range, candidates for laparoscopic cholecystectomy surgery, ASA class I and II, mallampati grade 1 or 2 , and TMD $>6 \mathrm{~cm}$.

Exclusion criteria were the history of smoking or drug abuse, history of common cold during the previous two weeks, patients with COPD, asthma, and convulsions and those known to be allergic to the study drugs.
We selected 200 patients, scheduled for laparoscopic cholecystectomy, and distributed them to one of the five defined groups using random numbers tables.

All patients underwent a fixed general anesthetic technique employing $2 \mathrm{mg} / \mathrm{kg}$ propofol, $0.2 \mathrm{mg} / \mathrm{kg}$ sufentanil and $0.5 \mathrm{mg} / \mathrm{kg}$ atracurium, followed by laryngoscopy and intubation in all patients.

ETT cuff was treated in the following order:

Group P simple gel (placebo) one $\mathrm{mL}$ applied,

Group T1 tramadol gel (2.5\%) one mL applied,

Group T2 tramadol gel (5\%) one mL applied,

Group L lidocaine gel (2\%) one mL applied,

And in Group C no drug was used.

Patients who needed greater than one attempt of laryngoscopy were excluded from the study.

To prepare $2.5 \%$ tramadol gel, $25 \mathrm{mg} / \mathrm{ml}$ of tramadol was dissolved in distilled water (double distillation) and the carbomer powder was slowly added to the drug solution at $40^{\circ} \mathrm{C}$ until the carbomer concentration reached to 1.5$2 \%$. Finally, the gel was cooled and kept refrigerated in a sealed container until use. To prepare the placebo gel, $1.5-2 \%$ carbomer without any drug was used.

After the intubation, the ETT cuff was filled with air with pressure equivalent to $25 \mathrm{cmH}_{2} \mathrm{O}$. Isoflurane gas was used to maintain anesthesia.

For all patients, surgery was performed by a single surgeon. At the end of anesthesia, all patients were reversed by $0.04 \mathrm{mg} / \mathrm{kg}$ neostigmine and $0.02 \mathrm{mg} / \mathrm{kg}$ atropine.

Patients were awakened and mouth suction was conducted with the pressure of less than 100 $\mathrm{cmH}_{2} \mathrm{O}$. The occurrence of bucking, coughing, laryngospasm and bronchospasm was noted and data were recorded. The patients were followed for the incidence of the sore throat using VAS at the first, sixth and twelfth hours after the extubation and findings were recorded. Patients with a VAS greater than 5 were treated with meperidine. 
Original Research

Table 1: Demographic features and the comparison of the incidence of cough in five studied groups

\begin{tabular}{lccccccc}
\multicolumn{1}{c}{ Variable } & Group P & Group T1 & Group T2 & Group L & Group C & $\begin{array}{c}\text { Test } \\
\text { Statistics }\end{array}$ & P-Value \\
\hline Age (mean \pm SD) & $43.29 \pm 9.02$ & $44.51 \pm 8.72$ & $45.36 \pm 6.73$ & $42.8 \pm 8.65$ & $42.87 \pm 7.68$ & 2.77 & 0.596 \\
\cline { 1 - 5 } Male [n (\%)] & $8(21.1)$ & $15(38.5)$ & $13(36.1)$ & $17(42.5)$ & $14(29.8)$ & & \\
\cline { 1 - 5 } Female [n (\%)] & $30(78.9)$ & $24(61.5)$ & $23(63.9)$ & $23(57.5)$ & $33(70.2)$ & & 0.295 \\
\cline { 1 - 5 } Cough [n (\%)] & $17(44.7)$ & $21(53.8)$ & $9(25)$ & $17(42.5)$ & $27(67.5)$ & 15.4 & 0.004 \\
\hline
\end{tabular}

\section{Sample Size:}

The sample size was considered to be 36 in each group with regard to $95 \% \mathrm{CI}$ and statistical power of $90 \%$ based upon an earlier study, in which the incidence of cough with and without lidocaine gel was equal to $\mathrm{P} 1=0.4$ and $=0.31$ )

(1).

Data analysis:

Data were analyzed using IBM SPSS

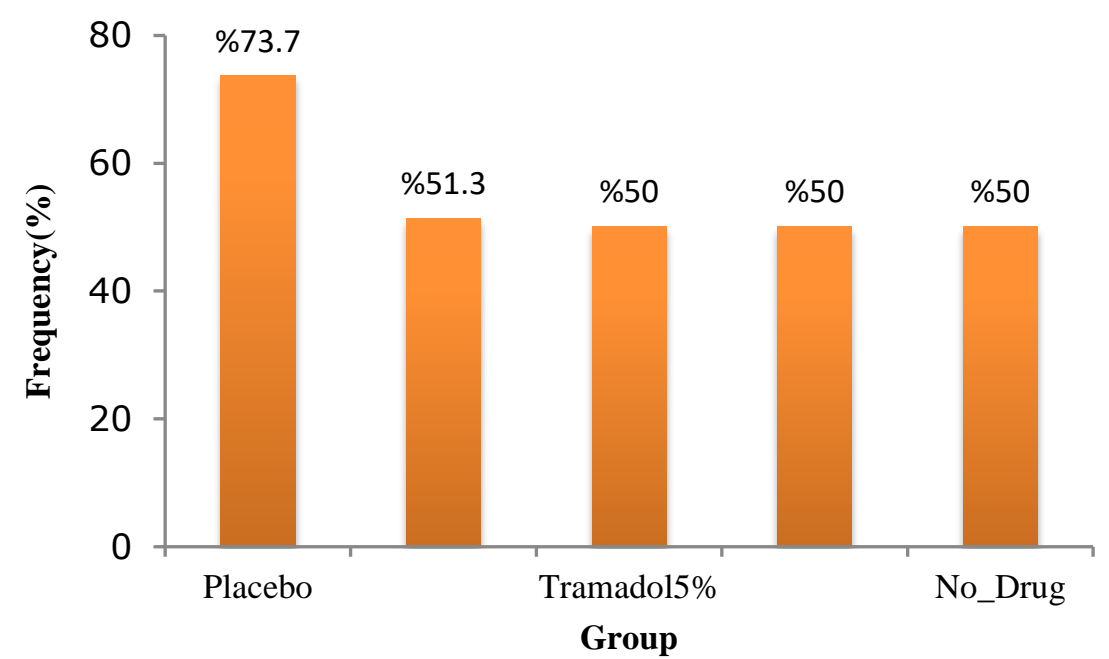

Statistics for Windows, Figure 1: Bucking incidence frequency in five studied groups of patients' candidate for laparoscopic Version 20.0. Armonk, cholecystectomy

NY: IBM Corp.). Chi-

square test was used to compare the qualitative variables between the two groups. For the quantitative dependent variables, one-way variance analysis (ANOVA) or Kruskal-Wallis test was used and in case of significance, the subsequent binary comparison tests were applied. The significance level of tests was considered as $\mathrm{p}<0.05$.

\section{RESULTS}

\section{Descriptive features:}

Two hundred patients participated in this study. The minimum sample size was 36 in each group based on the PASS2011, and to increase the accuracy of the results in some groups, more participants were enrolled.

38 patients (19\%) in the placebo group (Group P), 39 patients $(19.5 \%)$ in $2.5 \%$ tramadol group (Group T1), 36 patients (18\%) in 5\% tramadol group (Group T2), 40 patients (20\%) in the lidocaine group (Group L), and 47 patients (23.5\%) in no drug group (Group C).

Patients were between 22 to 60 y old $(43.71 \pm$ 8.18 years). One hundred thirty-three patients $(66.5 \%)$ were female and 67 patients $(33.5 \%)$ were male.

Table 1 shows no significant difference between the mean age and the gender of patients in five groups $(\mathrm{p}>0.05)$.

According to the results presented in Table 1, there was a significant difference between the 


\section{A comparative study of the effects of tramadol and lidocaine gel on complications}

incidence of coughing during extubation in five groups of patients $(\mathrm{p}<0.05)$.

A dramatic decrease in coughing was observed in the 5\% tramadol group. Although 5\% tramadol decreases the coughing clinically more than $2 \%$ lidocaine group, the difference was statistically not significant $(\mathrm{p}=0.108)$.

There was no significant difference between the frequency of bucking in $2.5 \%$ tramadol group and $5 \%$ tramadol group $(\mathrm{p}=0.897), 2.5 \%$ tramadol group and lidocaine group $(\mathrm{p}=0.9)$, $5 \%$ tramadol group and lidocaine group $(\mathrm{p}=$ 0.8) (Figure1).

There was no significant difference between the severity of sore throat at 1st, 6th, and 12th hours after extubation in between five groups ( $p>$ 0.05) (Figure 2).

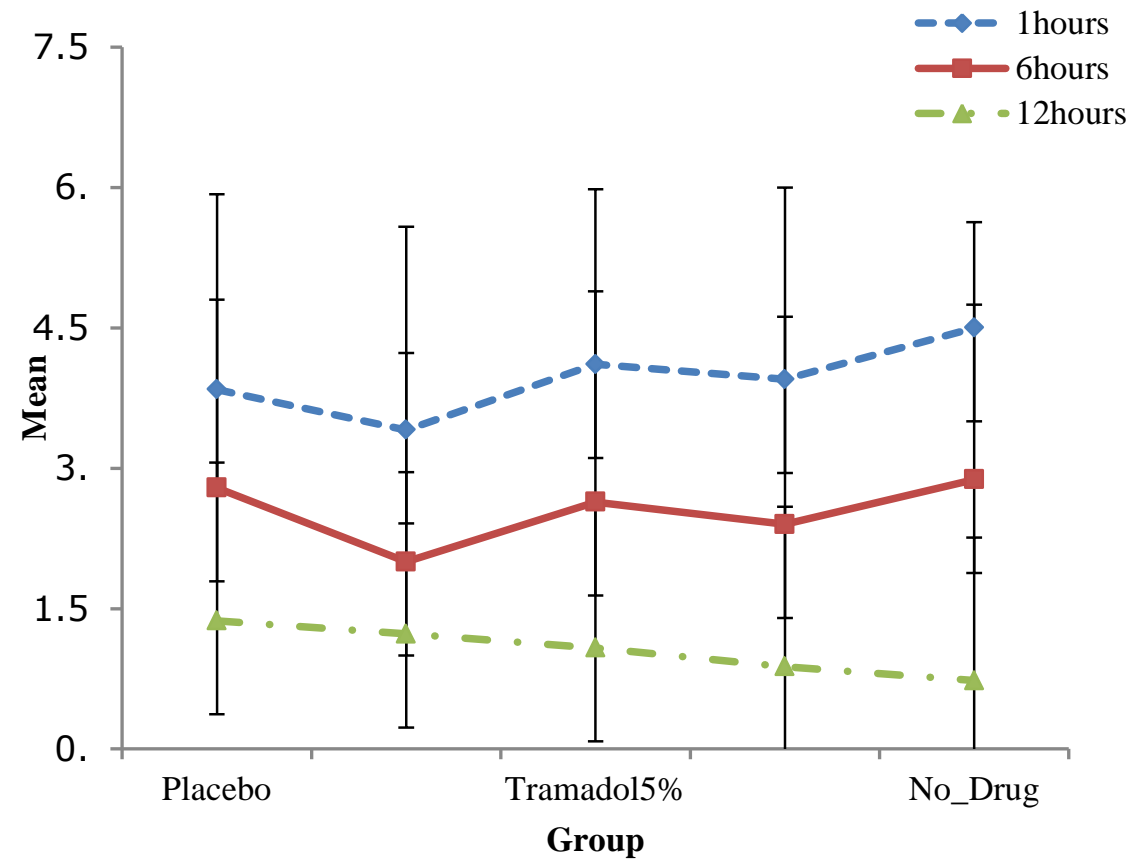

Figure 2: The average severity of sore throat in five studied groups

\section{DISCUSSION}

The results showed no significant difference between the mentioned groups in terms of age and gender of patients. In this study the effect of application of topical lidocaine gel and tramadol gel on ETT cuff on ETI related complications was evaluated. There was no significant difference between these five groups in terms of coughing, bucking, bronchospasm, laryngospasm and sore throat. The incidence of coughing was reduced in 5\% tramadol group, but the frequency of bucking was increased in placebo group.

According to the results of this experiment, a significant difference was observed between the ratios of coughing incidence in five groups of patients. So, $5 \%$ tramadol gel application led to a reduction in the incidence of coughing.

Sumathi et al. showed that the utilization of lidocaine gel in the ETI hadn't decreased the coughing incidence in comparison with the control group. This lack of consistency can be due to the variety of surgery, gel-forming substance, and extubation protocol. ${ }^{6}$ In our survey, the binary comparison revealed that bucking incidence in placebo gel consumers is more than all other groups.

The results of the present study emphasized the effect of active drug in the gel on reducing the incidence of bucking. Some researchers demonstrated that tramadol has local anesthetic and analgesic effects like lidocaine. ${ }^{9,10}$ 
Akbay et al. indicated that the pain of children with tramadol gel on ETT cuff for tonsillectomy was significantly lower than that of the normal saline group at $21 \mathrm{~h}$ and 7 days after surgery.

In Akbay and Heiba's studies, the positive effects of tramadol on reducing sore throat may be due to the systemic absorption of the drug in the high vascular region of inflamed tonsils. Tonsil's blood circulation is much greater than endotracheal mucosa and the tramadol dosage in this study was less than half of their used dosage. ${ }^{11-12}$ The results from other studies also confirm the possibility of systemic absorption. ${ }^{13,14}$

In some studies, lidocaine gel has been used for reducing sore throat caused by the ETI in comparison with gels treated with other drugs such as betamethasone, beclomethasone gels. They also concluded that treating the ETI cuff with lidocaine gel had no impact on the reduction of postoperative sore throat compared to the control group; ${ }^{6,15-16}$ even some of them have reported that the use of lidocaine gel can be worse. ${ }^{17}$

Mekhemar et al. obtained results in parallel with our survey and found that the use of 5\% lidocaine gel in the ETI cuff does not prevent sore throat, however, it is better than $10 \%$ lidocaine or normal saline spray. ${ }^{18}$

According to the results of this study, no significant difference was observed between the ratios of laryngospasm or bronchospasm incidence in five groups of patients' candidates for laparoscopic cholecystectomy surgery. In the literature, the rate of bronchospasm caused by the ETI has been mentioned to be $1 \% .^{19}$ It is similar to our study.

Since similar studies haven't compared the rate of bronchospasm incidence caused by the ETI treated with drugs such as tramadol and lidocaine, comparing the results of our study with other related studies was not possible.

\section{CONCLUSION}

According to the results of this study, application of $5 \%$ tramadol gel is effective in reducing the incidence of coughing caused by the endotracheal intubation, equivalent to the commonly used lidocaine gel. However, tramadol gel does not have an impact on the reduction of bucking, sore throat, bronchospasm, and laryngospasm caused by the endotracheal intubation.

\section{Funding:}

No funding for the present study.

Competing interests:

The authors declare that they have no competing interests.

\section{Authors' contribution:}

HF: Design and literature review

MY: Concept and performing the study

GM: Literature review, drafting

MR: Statistical analysis, drafting 


\section{A comparative study of the effects of tramadol and lidocaine gel on complications}

\section{REFERENCES}

1. Bagheri $\mathrm{H}$, Nourian $\mathrm{J}$, Ebrahimi $\mathrm{H}$, Maghsoud T, Atash S. Prevalence and specifications of postoperative sore throat following general endotracheal anesthesia in patients undergoing surgery. Journal of Knowledge \& Health. 2007;2:7-11.

2. Alavi S.M, Mahjoubifard M, Panahipour A.A. The effect of intracuff lidocaine on cough, bucking and laryngo-spasm during emergence from general anesthesia.Journal of Iranian Society Anaesthesiology and Intensive Care.2003:24:49-56.

[Free full text] DOl: https://doi.org/10.1371/journal.pone. 0136184

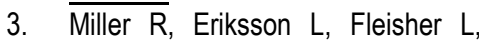
Wiener -Kronish J, Cohen N,Young W. Miller's Ansthesia .7th ed. Philadelphia Elsevier; 2010.

4. Yukioka H, Yoshimoto N, Nishimura $\mathrm{K}$, Fujimori M. Intravenous lidocaine as a suppressant of coughing during tracheal intubation. Anesth Analg. 1985;64:1189-92.

[PubMed]

5. Phani Krishna Y, Madhuri S.M.S, Madhuri R. Formulation and In vitro evaluation of tramadol hydrochloride gel. Int J S Res Sci. Tech. 2018; 4: 341-345.

[Free full text]

6. Sumathi PA, Shenoy T, Ambareesha M, Krishna HM. Controlled comparison between betamethasone gel and lidocaine jelly applied over tracheal tube to reduce postoperative sore throat, cough, and hoarseness of voice. $\mathrm{Br}$ J Anaesth. 2008;100:215-8. [PubMed] [Free full text] DOl: 10.1093/bja/aem341

7. Rashwan $\mathrm{S}$, Abdelmawgoud $\mathrm{A}$, Badawy AA. Effect of tramadol gargle on postoperative sore throat: A double blinded randomized placebo controlled study. Egyptian J Anaesth. 2014;30(3):235-239
[Free full text] DOI:

https://doi.org/10.1016/j.egja.2014.0 1.010

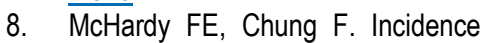
and severity of postoperative sore throat: a randomized comparison of Glidescope with Macintosh laryngoscope. Anaesthesia. 1999:54:444-53.

[PubMed] [Free full text] DOI: 10.1046/.j.1365-2044.1999.00780.x

9. Wahhabi S, Moradkhani M, Farzan $B$, Zarei F, Akhlaghi $Z$. The comparison of tramadol and lidocaine anesthetic features in small surgeries under local anesthesia by subcutaneous block. Scientific Research Journal of Lorestan University of Medical Sciences. 2008;1:31-5.

10. Heiba $\mathrm{MH}$, Atef $\mathrm{A}$, Mosleh $\mathrm{M}$, Mohamed R, El-Hamamsy $M$. Comparison of peritonsillar infiltration of tramadol and lidocaine for the relief of post-tonsillectomy pain. J Laryngol Otol. 2012;126:1138-41.

[PubMed] [Free full text] DOl: 10.1017/S0022215112002058

11. Akbay BK, Yildizbas S, Guclu E, Yilmaz S, Iskender A, Ozturk $O$. Analgesic efficacy of topical tramadol in the control of postoperative pain in children after tonsillectomy. J Anesth. 2010;24:705-8.

[PubMed] DOI: $10.1007 / \mathrm{s} 00540-$ 010-0978-2

12. Kargi E, Isikdemir A, Tokgoz H, Erol $B$, Isikdemir $F$, Hanci $V$, et al. Comparison of local anesthetic effects of tramadol with prilocaine during circumcision procedure. Urology. 2010;75:672-5.

[PubMed] DOl:

10.1016/j.urology.2009.06.108

13. Lin BF, Ju DT, Cherng $\mathrm{CH}$, Hung NK, Yeh CC, Chan SM, et al. Comparison between intraoperative fentanyl and tramadol to improve quality of emergence. J Neurosurg Anesthesiol. 2012;24:127-32. [PubMed] DOI: 10.1097/ANA.0b013e31823c4a24

14. Maruyama K, Sakai H, Miyazawa H, Toda N, linuma $Y$, Mochizuki N, et al. Sore throat and hoarseness after total intravenous anaesthesia. $\mathrm{Br} \mathrm{J}$ Anaesth. 2004;92:541-3. [PubMed] [Free full text]

15. Soltani HA, Aghadavoudi O. The effect of different lidocaine application methods on postoperative cough and sore throat. J Clin Anesth. 2002;14:15-8. [PubMed] [Free full text] DOl: 10.1016/s0952-8180(01)00344-0

16. Banihashem $N$, Alijanpour $E$, Hasannasab B, Zarei A. Prophylactic Effects of Lidocaine or Beclomethasone Spray on PostOperative Sore Throat and Cough after Orotracheal Intubation. Iran J Otorhinolaryngol. 2015;27:179-84. [PubMed] [Free full text]

17. Kori K, Muratani T, Tatsumi S, Minami T. Influence of endotracheal tube cuff lubrication on postoperative sore throat and hoarseness [Article in Japanese]. Masui. 2009;58:342-5.

[PubMed]

18. Mekhemar NA, El-Agwany AS, Radi WK, El-Hady SM. Comparative study between benzydamine hydrochloride gel, lidocaine $5 \%$ gel and lidocaine $10 \%$ spray on endotracheal tube cuff as regards postoperative sore throat. Braz J Anesthesiol. 2016;66:242-8. [PubMed] [Free full text] DOI: 10.1016/j.bjane.2014.09.007

19. Miller R, Eriksson L, Fleisher L, Wiener -Kronish J,Young W. Miller's Ansthesia .7th ed. Philadelphia Elsevier; 2009. 